Evaluation of volume change in rectum and bladder during application of image-guided radiotherapy for prostate carcinoma

J. A. Luna' and J. I. Rojas'

Citation: AIP Conference Proceedings 1753, 080022 (2016); doi: 10.1063/1.4955392

View online: http://dx.doi.org/10.1063/1.4955392

View Table of Contents: http://aip.scitation.org/toc/apc/1753/1

Published by the American Institute of Physics 


\title{
Evaluation of Volume Change in Rectum and Bladder During Application of Image-Guided Radiotherapy for Prostate Carcinoma
}

\author{
J. A. Luna ${ }^{1, \text { a) }}$ and J. I. Rojas ${ }^{2,3, \text { b) }}$ \\ ${ }^{1}$ Departamento de Física, Universidad Nacional de Costa Rica, Heredia, Costa Rica. \\ ${ }^{2}$ Centro Médico Radioterapia Siglo XX1, La Uruca, Costa Rica. \\ ${ }^{3}$ PROXTRONICS CR, Ltda, Heredia, Costa Rica. \\ a)yosimoon13@hotmail.com \\ b)isaac.rojas@siglo21.cr
}

\begin{abstract}
All prostate cancer patients from Centro Médico Radioterapia Siglo XXI receive Volumetric Modulated Arc Therapy (VMAT). This therapy uses image-guided radiotherapy (IGRT) with the Cone Beam Computed Tomography (CBCT). This study compares the planned dose in the reference CT image against the delivered dose recalculate in the CBCT image. The purpose of this study is to evaluate the anatomic changes and related dosimetric effect based on weekly $\mathrm{CBCT}$ directly for patients with prostate cancer undergoing volumetric modulated arc therapy (VMAT) treatment. The collected data were analyzed using one-way ANOVA.
\end{abstract}

\section{INTRODUCTION}

Prostate cancer is the second leading cause of cancer deaths in men only behind lung cancer [1].The database GLOBOCAN 2002 from the International Agency for Research on Cancer, estimates 515 new cases and 327 deaths per year in Costa Rica [1].

The Volumetric Modulated Arc Therapy (VMAT) is one strategy to perform a good treatment for this kind of cancer. VMAT allows to physician outlines the patient's tumor volume and keeps protecting organs at risk (OARs). However, when this type of complex treatment is used, it is necessary to use Image-Guided Radiotherapy (IGRT), in order to assure accuracy in daily set up.

There are different ways to perform IGRT, but we are going to focus on Cone Beam Computed Tomography $(\mathrm{CBCT})$, which is performed on each patient prior to delivery the treatment.

In the evaluation of the treatment plan, the following aspects are important:

1. The planning target volume (PTV) must be fully covered by the $95 \%$ isodose [2].

2. Femur, bladder, and rectum are organs at risk that should be within the limit dose-volume constraints [2].

Anatomical changes along the prostate treatment have to be taken into account. For instance, bladder and rectum can be in any state between filled and emptied.

Most cancer centers use full-bladder protocols for radiation therapy on patients with prostate cancer [3]. However, it is acknowledged that through the course of radiation therapy, bladder filling may vary in a random manner. These variations are more common in elderly patients or in those with irritating urinary symptoms. The filling of the bladder may also affect the position of the prostate significantly and it can have a negative impact on the accuracy of the radiotherapy [3].

Besides, the use of the VMAT planning is the best way to treat the prostate cancer because this technique delivers the maximum dose of the planning target volume (PTV), while sparing organs at risk. It produces more conformal dose distributions that can reduce normal tissue toxicity. Also E. Mellon et al have observed VMAT for 
treatment prostate cancer reduces beam-on time and the amount of radiation delivered (monitor units), relative to 7 to 9-field, step - and shoot IMRT [4].

The purpose of this study is to evaluate the anatomic changes and related dosimetric effect based on weekly $\mathrm{CBCT}$ directly for patients with prostate cancer undergoing volumetric modulated arc therapy (VMAT) treatment.

\section{MATERIALS AND METHODS}

\section{Patient Characteristics and Planning}

This study was performed in the Centro Medico Radioterapia Siglo XXI located in La Uruca, Costa Rica. We retrospectively reviewed 8 patients with adenocarcinoma of the prostate treated between January 2015 and November 2015. Patients were treated using volumetric modulated arc therapy (VMAT). Daily image was performed using the CBCT. However we used weekly CBCT images to execute this study to simplify the collected data.

\section{CBCT Imaging}

VMAT plans were delivered on Trilogy Linac (Varian, Medical System, USA), which is integrated with an onboard kV-CBCT. CBCT images were acquired at the first treatment day (CBCT1) with patients in the treatment position prior to radiation delivery, and then performed day after day. The acquisition parameters were $125 \mathrm{kV}, 80$ $\mathrm{mA}, 50 \mathrm{~ms}$ per projection with F0 filter.

\section{Volumetric and Dosimetric Evaluations}

All initial CT data were exported to Eclipse TPS through DICOM service. All CT imaging was scanned from the level of the third lumbar vertebrate to the middle of the femurs for treatment simulation on a GE Lightspeed CT using a slice thickness of $5 \mathrm{~mm}$.

Volumetric changes and dosimetric results recalculated on the CBCT images were investigated using the Eclipse Treatment Planning for External Beam System (TPS) (clinical version 13.0; Varian Medical System, USA).

The OARs were manually contoured in every $\mathrm{CBCT}$ data set to evaluate the variations.

\section{Statistical Analysis}

Descriptive statistics were calculated to characterize the dosimetric and volumetric changes of the OARs. Differences between the planned dose in the initial CT and recalculated dose based on weekly CBCTs were analyzed using one-way ANOVA. When an overall significant difference was observed, the LSD Fisher test was used to determine which pairwise comparisons differed [5].All statistical analyses were conducted with InfoStat software (Grupo InfoStat, Cordoba, 2011). Differences were considered statistically significant when the probability value $\mathrm{p}<0.05[5]$.

\section{RESULTS}

Dosimetric differences resulting from volume changes and geometrical errors are shown on table 1. 
TABLE 1.Dosimetric differences between planned dose in the CT image and delivered dose in the CBCT image.

\begin{tabular}{cccccc}
\hline Rectum & C T & CBCT1 & CBCT1 & CBCT1 & P \\
\hline $\mathrm{D}_{\text {max }}(\mathrm{cGy})$ & $6384 \pm 135$ & $6412 \pm 144$ & $6371 \pm 160$ & $6449 \pm 164$ & 0.944 \\
$\mathrm{D}_{\text {mean }}(\mathrm{cGy})$ & $2594 \pm 127$ & $3246 \pm 112$ & $3208 \pm 113$ & $3090,15 \pm 95$ & 0.0327 \\
$\mathrm{D}_{15}(\mathrm{cGy})$ & $4274 \pm 106$ & $4861 \pm 131$ & $4859 \pm 143$ & $4503 \pm 129$ & 0.2787 \\
$\mathrm{D}_{25}(\mathrm{cGy})$ & $33185 \pm 163$ & $4236 \pm 127$ & $4290 \pm 133$ & $4015 \pm 121$ & 0.0724 \\
$\mathrm{D}_{35}(\mathrm{cGy})$ & $2330 \pm 166$ & $3083 \pm 132$ & $2881 \pm 132$ & $2479 \pm 147$ & 0.1137 \\
$\mathrm{D}_{50}(\mathrm{cGy})$ & $2736 \pm 164$ & $3732 \pm 143$ & $3796 \pm 144$ & $3186 \pm 155$ & 0.0943 \\
$\left.\mathrm{~V}_{\text {olume }(\mathrm{cm}}{ }^{3}\right)$ & $143 \pm 6$ & $96 \pm 4$ & $111 \pm 3$ & $114 \pm 3$ & 0.0137 \\
\hline Bladder & $\mathbf{C T}$ & $\mathbf{C B C T 1}$ & $\mathbf{C B C T 1}$ & $\mathbf{C B C T 1}$ & $\mathbf{P}$ \\
\hline $\mathrm{D}_{\text {max }}(\mathrm{cGy})$ & $6495 \pm 150$ & $6344 \pm 214$ & $6545 \pm 155$ & $6556 \pm 153$ & 0.7617 \\
$\mathrm{D}_{\text {mean }}(\mathrm{cGy})$ & $3061 \pm 104$ & $3690 \pm 119$ & $3369 \pm 177$ & $3316 \pm 93$ & 0.4573 \\
$\mathrm{D}_{15}(\mathrm{cGy})$ & $4619 \pm 120$ & $5171 \pm 232$ & $4905 \pm 160$ & $5394 \pm 150$ & 0.3581 \\
$\mathrm{D}_{25}(\mathrm{cGy})$ & $3856 \pm 123$ & $4278 \pm 190$ & $4447 \pm 195$ & $4552 \pm 106$ & 0.6837 \\
$\mathrm{D}_{35}(\mathrm{cGy})$ & $3359 \pm 138$ & $3596 \pm 181$ & $3934 \pm 181$ & $3837 \pm 113$ & 0.8102 \\
$\mathrm{D}_{50}(\mathrm{cGy})$ & $2817 \pm 139$ & $3210 \pm 15$ & $3155 \pm 210$ & $2989 \pm 158$ & 0.8925 \\
$\left.\mathrm{~V}_{\text {olume }}(\mathrm{cm})^{3}\right)$ & $323 \pm 15$ & $255 \pm 15$ & $178 \pm 13$ & $255 \pm 9$ & 0.0695 \\
\hline
\end{tabular}

\section{DISCUSSION}

Several studies have demonstrated that interfraction and intrafraction variations in organ filling result in corresponding changes in the distance between the bladder and the rectum [3].Therefore, the planned dose has been a poor predictor of the delivered dose to the rectum and bladder in patients receiving radiotherapy for localized prostate cancer as consequence of instability in rectum and bladder movement and filling [3]. Delivered doses can be more precisely assessed using daily CBCT scans acquired during treatment [6]. Daily CBCT images give the chance to observe the accurate target localization during the radiotherapy, offering less toxicity to the patients [6].

These $\mathrm{CBCT}$ images were directly applied to study the volumetric changes and dosimetric results of 8 prostate carcinoma patients underwent VMAT treatment.

Our study has shown that the delivered dose to the bladder was not showing significant differences with that planned dose because variables $D_{\max }, D_{\text {mean }}, D_{50}, D_{35}, D_{25}$, and $D_{15}$ show a probability value overhead 0.05 .

For dose-volume constraints, the bladder met the limits of the clinic protocol acceptance criteria for radiotherapy, see table 2 . 
TABLE 2.Dose volume constraints for each organ. [7]

\begin{tabular}{ccc}
\hline $\begin{array}{c}\mathbf{D}_{\text {volume }} \\
(\mathbf{\%})\end{array}$ & $\begin{array}{c}\text { Bladder Constraints } \\
(\mathbf{c G y})\end{array}$ & $\begin{array}{c}\text { Rectum Constraints } \\
(\mathbf{c G y})\end{array}$ \\
\hline 15 & $<8000$ & $<7500$ \\
25 & $<7500$ & $<7000$ \\
35 & $<7000$ & $<6500$ \\
50 & $<6500$ & $<6000$ \\
\hline
\end{tabular}

Changes in the bladder volume are significantly different in the CT image and in the CBCT images because the probability value $(\mathrm{p}=0.0327)$ is less than 0.05 . These changes happen due to incorrect bladder filling, and this may vary in a systematic manner. Additionally all elderly patients or with irritating urinary symptoms find it difficult to maintain a full bladder during radiotherapy.

In the rectum case, the changes in the volume are also significant due to the probability value $(\mathrm{p}=0.0137)$.

For the dose-volume constraints, the rectum met the limits of the clinic plan acceptance criteria for radiotherapy.

As it is known that new plans performed using CBCT scans can give more accurate assumptions of the overall delivered doses. Clinical application of IGRT for prostate cancer has shown significant improvement in terms of tumor control and low toxicity profile [6].

\section{PRELIMINARY CONCLUSION}

This study showed that CBCT data are suitable to provide an approach in adaptive radiotherapy, which may be useful in the clinical routine to respond adequately to daily anatomy variations of the patient to optimize the therapeutic success and the protection of the organs at risk.

The dose-volume constraints of the organ at risk met the limits of the clinic plan acceptance criteria for radiotherapy.

\section{ACKNOWLEDGEMENTS}

This work has been supported by the Centro Medico Radioterapia Siglo XXI (San José, Costa Rica), $21^{\text {st }}$ Century Oncology Corporate, Ministerio de Ciencia, Tecnología y Telecomunicaciones (MICITT), and Maestría en Física Médica of the Universidad Nacional de Costa Rica (UNA).

\section{REFERENCES}

1. J. Ferlay, H.R. Shin, F. Bray, D. Forman, C.D. Mathers, et at, Int J Cancer.127, 2893(2010).

2. H.M. Chairman, J ICRU.10, 1(2010).

3. V. Moissenko, M. Liu, S. Kristensen, G. Gerlowits and E. Berthelet. Am. Coll. Med. Phys.8, 55(2007).

4. E. Mellon. K. Javedan, J. Strom, E. Moros, M.C. Biagioli, et at, Pract Radiat Oncol.5, 11 (2015).

5. G. Snedecor, and W.G. Cochran, Statistic methods. (Lowa State University Press, United States of America, 1967), p.321.

6. S. Song, K. Yenice, M. Kopec, and S. Liauw, Radiother Oncol.103, 270(2012).

7. B.M. Marks, E.D. York, A. Jackson, R.K. Ten Haken, L.S. Cuestine, A, et at, Int J Radiat Oncol Biol Phys.76,10(2010), available at

http://www.ncbi.nlm.nih.gov/pmc/articles/PMC4041542/ 